\title{
CONTINUED FRACTIONS AND TOTALLY MONOTONE SEQUENCES
}

\author{
BY
}

\author{
H. S. WALL
}

1. Introduction. A sequence $c_{0}, c_{1}, c_{2}, \cdots$ of real numbers is called totally monotone if $\Delta^{m} c_{n} \geqq 0,(m, n=0,1,2, \cdots)$, where

$$
\Delta^{m} c_{n}=c_{n}-C_{m, 1} c_{n+1}+C_{m, 2} c_{n+2}-\cdots+(-1)^{m} C_{m, m} c_{n+m} .
$$

Hausdorff( $\left.{ }^{1}\right)$ showed that for every totally monotone sequence $c_{0}, c_{1}, c_{2}, \ldots$ there exists (essentially uniquely) a monotone nondecreasing real function $\phi(u), 0 \leqq u \leqq 1$, such that

$$
c_{n}=\int_{0}^{1} u^{n} d \phi(u), \quad n=0,1,2, \cdots
$$

Conversely, if $\phi(u)$ is a monotone nondecreasing bounded real function on the interval $0 \leqq u \leqq 1$, then $\Delta^{m} c_{n}=\int_{0}^{1}(1-u)^{m} u^{n} d \phi(u) \geqq 0, m, n=0,1,2, \cdots$, so that $c_{0}, c_{1}, c_{2}, \cdots$ is totally monotone.

In case the function $\phi(u)$ has an infinity of points of increase in the interval $0 \leqq u \leqq 1$, then the corresponding sequence is a special Stieltjes moment sequence, and accordingly there is a Stieltjes continued fraction $\left({ }^{2}\right)$

$$
b_{1} / 1+b_{2} x / 1+b_{3} x / 1+\cdots,
$$

in which the numbers $b_{1}, b_{2}, b_{3}, \cdots$ are real and positive, which corresponds to the power series

$$
c_{0}-c_{1} x+c_{2} x^{2}-\cdots .
$$

On the other hand, if $\phi(u)$ has but a finite number of points of increase, then the series (1.2) represents a rational function of $x$ and the continued fraction terminates.

The main problem which we have solved in the present paper is as follows: to find necessary and sufficient conditions upon the numbers $b_{1}, b_{2}, b_{3}, \ldots$ in the continued fraction (1.1) in order that the coefficients $c_{0}, c_{1}, c_{2}, \cdots$ in the corresponding power series (1.2) shall form a totally monotone sequence. The re-

Presented to the Society February 24, 1940; received January 30, 1940. This paper is dedicated to Edward Burr Van Vleck on the occasion of his seventy-seventh birthday, June 7, 1940.

(1) F. Hausdorff, Ueber das Momenten problem für ein endliches Interval, Mathematische Zeitschrift, vol. 16 (1923), pp. 220-248.

(2) T. J. Stieltjes, Recherches sur les fractions continues, Oeuvres, vol. 2, pp. 402-566. We have made the substitution of $x$ for $1 / z$, and have put $b_{1}=1 / a_{1}, b_{n}=1 / a_{n} a_{n-1}, n \geqq 2$, in the series and continued fraction used by Stieltjes. 
sult is very simple, namely: the sequence $c_{0}, c_{1}, c_{2}, \cdots$ is totally monotone if and only if there exist real numbers $g_{0}, g_{1}, g_{2}, \cdots$ such that $0 \leqq g_{n} \leqq 1$, $n=0,1,2, \cdots$, and such that

$$
\begin{aligned}
& c_{0}-c_{1} x+c_{2} x^{2}-c_{3} x^{3}+\cdots \sim g_{0} / 1+g_{1} x / 1+\left(1-g_{1}\right) g_{2} x / 1 \\
&+\left(1-g_{2}\right) g_{3} x / 1+\cdots,
\end{aligned}
$$

it being agreed that the continued fraction $\left(^{3}\right)$ shall terminate with the first identically vanishing partial quotient.

If $c_{0}, c_{1}, c_{2}, \cdots$ is totally monotone, the function $f(x)$ represented by the power series (1.2) is analytic for $|x|<1$. Let $M(f)=1$.u.b. $|x|<1|f(x)|$. We show that $M(f)$ is finite if and only if the series $c_{0}+c_{1}+c_{2}+\cdots$ converges, and establish the equality

$$
M(f)=c_{0}+c_{1}+c_{2}+\cdots .
$$

We also characterize the class $E$ of these "moment generating functions" which are bounded in the unit circle (a) in terms of the Stieltjes integral representation of $f(x)$, and (b) in terms of the continued fraction representation of $f(x)$. It is shown that if $f(x) \varepsilon E, M(f) \leqq 1$, then the functions defined by the algorithm of $\operatorname{Schur}\left({ }^{4}\right)$, namely:

$$
f_{n+1}=\frac{1}{x} \frac{t_{n}-f_{n}}{1-t_{n} f_{n}}, \quad t_{n}=f_{n}(0),
$$

$n=0,1,2, \cdots, f_{0}=f$, are all in $E$ and have moduli not exceeding 1 for $|x|<1$.

2. An operation on continued fractions. We shall use the symbol " " between a power series $P(x)$ and a continued fraction $K(x)$ to indicate that the power series expansion of the $n$th approximant of $K(x)$ agrees term by term with $P(x)$ for more and more terms as $n$ is increased, or becomes identical with $P(x)$ from and after some value of $n$. The basic theorem of the paper is

TheOREM 2.1. If $g_{1}, g_{2}, g_{3}, \cdots$ are any real or complex numbers, and $P(x)$ is a power series in ascending powers of $x$ such that

$$
P(x) \sim 1+g_{1} x / 1+\left(1-g_{1}\right) g_{2} x / 1+\left(1-g_{2}\right) g_{3} x / 1+\cdots,
$$

then

$$
\frac{1+x}{P(x)} \sim 1+\left(1-g_{1}\right) x / 1+g_{1}\left(1-g_{2}\right) x / 1+g_{2}\left(1-g_{3}\right) x / 1+\cdots
$$

( $\left.{ }^{3}\right)$ Continued fractions of this form were first treated by E. B. Van Vleck, in a paper entitled $O n$ the convergence and character of the continued fraction $a_{1} z / 1+a_{2} z / 1+a_{3} z / 1+\cdots$, these Transactions, vol. 2 (1901), pp. 476-483.

(4) J. Schur, Ueber Potenzreihen, die im Innern des Einheitskreises beschränkt sind, Journal für die reine und angewandte Mathematik, vol. 147 (1916), pp. 205-232, and vol. 148 (1917), pp. 122-145. 
Proof. Let $A_{n}(x) / B_{n}(x), A_{n}^{*}(x) / B_{n}^{*}(x)$ be the $n$th approximants of the continued fractions in (2.1) and (2.2), respectively. Then we have the relations (2.3) $A_{n}(x)=g_{n} x B_{n-1}^{*}(x)+B_{n}^{*}(x), \quad(1+x) B_{n}(x)=g_{n} x A_{n-1}^{*}(x)+A_{n}^{*}(x)$, $\left(n=0,1,2, \cdots, g_{0}=1, A_{-1}^{*}=1, B_{-1}^{*}=0\right)$. These may be verified directly for $n=0,1$. Assuming that the first is true for $n \leqq m, m \geqq 1$, we then have

$$
\begin{aligned}
A_{m+1}(x) & =A_{m}(x)+g_{m+1}\left(1-g_{m}\right) x A_{m-1}(x) \\
& =g_{m} x B_{m-1}^{*}(x)+B_{m}{ }^{*}(x)+g_{m+1}\left(1-g_{m}\right) x\left[g_{m-1} x B_{m-2}^{*}(x)+B_{m-1}^{*}(x)\right] .
\end{aligned}
$$

Since $g_{m-1}\left(1-g_{m}\right) x B_{m-2}{ }^{*}(x)=B_{m}^{*}(x)-B_{m-1}{ }^{*}(x)$, we then have

$$
\begin{aligned}
A_{m+1}(x) & =g_{m+1} x B_{m}{ }^{*}(x)+\left[B_{m}{ }^{*}(x)+g_{m}\left(1-g_{m+1}\right) x B_{n^{-1}}(x)\right] \\
& =g_{m+1} x B_{m}{ }^{*}(x)+B_{m+1}{ }^{*}(x),
\end{aligned}
$$

so that the first relation $(2.3)$ is true for $n=m+1$, and therefore, by mathematical induction, for all $n$. The second relation (2.3) may be proved in a similar way.

On multiplying the first relation $(2.3)$ by $A_{n-1}^{*}(x)$, the second by $B_{n-1}^{*}(x)$, and then subtracting, we find that the power series expansion in ascending powers of $x$ of the rational function

$$
A_{n-1}^{*}(x) / B_{n-1}^{*}(x)-\frac{1+x}{A_{n}(x) / B_{n}(x)}
$$

begins with the $(n-1)$ th or a higher power of $x$. It follows immediately that the correspondence (2.1) implies the correspondence (2.2).

This theorem may be thrown into the following form:

THEOREM 2.2. Let $c_{0} \neq 0$, and

$$
\begin{aligned}
c_{0}-c_{1} x+c_{2} x^{2}-\cdots \sim c_{0} / 1+g_{1} x / 1+(1- & \left.g_{1}\right) g_{2} x / 1 \\
& +\left(1-g_{2}\right) g_{3} x / 1+\cdots .
\end{aligned}
$$

Then

$$
\begin{aligned}
\Delta c_{0}-\Delta c_{1} x+\Delta c_{2} x^{2}-\cdots \sim \Delta c_{0} / 1+g_{1}(1 & \left.-g_{2}\right) x / 1 \\
& +g_{2}\left(1-g_{3}\right) x / 1+\cdots .
\end{aligned}
$$

Proof. Let $c_{0} / P(x)=c_{0}-c_{1} x+c_{2} x^{2}-\cdots$ in Theorem 2.1. This gives at once

$$
\begin{aligned}
c_{0}+\left(c_{0}-c_{1}\right) x-\left(c_{1}-c_{2}\right) x^{2}+\cdots \sim c_{0}+c_{0}\left(1-g_{1}\right) x / 1 \\
\\
\quad+g_{1}\left(1-g_{2}\right) x / 1+\cdots .
\end{aligned}
$$

On removing the constant term $c_{0}$ from the series and from the continued fraction, and then dropping a factor $x$, the correspondence (2.5) results. We note for future reference that 


$$
\Delta c_{0}=c_{0}\left(1-g_{1}\right) \text {. }
$$

By means of these theorems we have enlarged by one the small list of known operations on continued fractions.

The next theorem makes the transformation available for a large class of continued fractions.

TheOREM 2.3. Let $A_{n}(x) / B_{n}(x)$ be the nth approximant of the continued fraction

$$
1+a_{1} x / 1+a_{2} x / 1+a_{3} x / 1+\cdots,
$$

and let $c \neq 0$ be any number such that $A_{n}(-c) \neq 0,(n=1,2,3, \cdots)$. Put

$$
g_{n}=a_{n} c A_{n-2}(-c) / A_{n-1}(-c),
$$

$n=1,2,3, \cdots, A_{-1}(-c)=1$. Then the continued fraction (2.7) takes the form

$$
1+\frac{g_{1}(x / c)}{1}+\frac{\left(1-g_{1}\right) g_{2}(x / c)}{1}+\frac{\left(1-g_{2}\right) g_{3}(x / c)}{1}+\cdots .
$$

Proof. One may verify immediately that $a_{1}=g_{1} / c, a_{n}=g_{n}\left(1-g_{n-1}\right) / c$, $n=2,3,4, \cdots$, when the $g_{n}$ 's are given by $(2.8)$.

We remark in passing that if $a_{n} \neq 0$ in (2.7), and $P(x)$ is the power series corresponding to (2.7), then one may apply Theorem 2.1 to obtain the formulas given by Stieltjes $\left({ }^{5}\right)$ for the continued fraction corresponding to $1 / P(x)$. In fact:

$$
\begin{aligned}
\frac{1+(x / c)}{P(x)} \sim 1 & +\frac{\left(1-g_{1}\right)(x / c)}{1}+\frac{g_{2}\left(1-g_{1}\right)(x / c)}{1}+ \\
+ & \frac{g_{3}\left(1-g_{2}\right)(x / c)}{1}+\cdots
\end{aligned}
$$

We may allow $c$ to become infinite and the correspondence will be maintained provided the coefficients of $x$ in the continued fraction have limits which are finite and not 0 . Accordingly we obtain this theorem:

THEOREM 2.4. If the power series $P(x)$ has a corresponding continued fraction (2.7) in which $a_{n} \neq 0, n \geqq 1$, then $1 / P(x)$ will have a corresponding continued fraction of the same form provided $A_{2 n}(x)$, the numerator of the $2 n t h$ approximant of (2.7), is of degree $n$ for $n=0,1,2, \cdots$.

The condition of the theorem is met when the $a_{n}$ 's are real and positive, which is the case with which Stieltjes was concerned. If one evaluates the limits for $c=\infty$ of the coefficients of $x$ in the continued fraction of (2.9), he will find that the result agrees with that found by Stieltjes.

(5) O. Perron, Die Lehre von den Kettenbrüchen, 1st edition, pp. 334-335. 
3. A uniform convergence theorem. In a recent $\left(^{6}\right)$ paper Scott and Wall proved a theorem which may be stated in the following form:

Theorem 3.1. If $g_{1}, g_{2}, g_{3}, \cdots$ are real numbers such that $0<g_{1}<1$, $0 \leqq g_{n}<1, n>1$, and $x_{1}, x_{2}, x_{3}, \cdots$ are functions of any variables, then the continued fraction

$$
\frac{1}{1}+\frac{\left(1-g_{1}\right) g_{2} x_{1}}{1}+\frac{\left(1-g_{2}\right) g_{3} x_{2}}{1}+\frac{\left(1-g_{3}\right) g_{4} x_{3}}{1}+\cdots
$$

converges uniformly for $\left|x_{n}\right| \leqq 1, n=1,2,3, \cdots$. The denominators of all the approximants are nonzero in this domain. Let $G$ denote the value of the continued fraction. Then

$$
|G| \leqq \frac{1}{g_{1}}\left\{1-\frac{1}{1+\sum_{1}^{\infty} \frac{g_{1} g_{2} \cdots g_{n}}{\left(1-g_{1}\right)\left(1-g_{2}\right) \cdots\left(1-g_{n}\right)}}\right\}
$$

if $\left|x_{n}\right| \leqq 1, n \geqq 1$; and $G$ is equal to the expression on the right if $x_{n}=-1, n \geqq 1$.

We shall digress momentarily at this point to discuss two theorems given by Perron on page 262 of his book. The first of these may, with no essential loss in generality, be stated as follows:

THEOREM 3.2. If the elements of the continued fraction

$$
1 / 1+a_{2} / 1+a_{3} / 1+a_{4} / 1+\cdots
$$

are functions of any variables, then the continued fraction converges uniformly over the domain characterized by the inequalities

$$
\left|a_{n}\right| \leqq\left(p_{n}-1\right) / p_{n} p_{n-1}, \quad n=2,3,4, \cdots,
$$

where $p_{1}, p_{2}, p_{3}, \cdots$ are any real constants greater than 1 for which the series

$$
\sum_{1}^{\infty}\left(p_{1}-1\right)\left(p_{2}-1\right) \cdots\left(p_{n}-1\right)
$$

is divergent.

Perron then says: "Ein bemerkenswerter Spezialfall unseres allgemeinen Kriteriums ist" and then proves a theorem, attributed to Van Vleck, which may be stated as follows:

THEOREM 3.3. If $g_{1}, g_{2}, g_{3}, \cdots$ are real numbers such that $0<g_{n}<1, n \geqq 1$, and $x_{1}, x_{2}, x_{3}, \cdots$ are functions of any variables, then the continued fraction (3.1) converges uniformly for $\left|x_{n}\right| \leqq 1, n \geqq 1$.

$\left.{ }^{(}\right)$W. T. Scott and H. S. Wall, $A$ convergence theorem for continued fractions, these Transactions, vol. 47 (1940), pp. 155-172. 
It is a strange fact that the second theorem is more general than the first. To see this, put $a_{n}=g_{n}\left(1-g_{n-1}\right) x_{n}, n \geqq 2 ; p_{n}=1 /\left(1-g_{n}\right), n \geqq 1$, and the second theorem reduces to the first minus the requirement on the series (3.3).

It should be added that Theorem 3.3 is related to but quite different from the theorem which Van Vleck proved $\left({ }^{7}\right)$. He gave preference to the continued fraction $1 / 1+g_{1} x_{0} / 1+\left(1-g_{1}\right) g_{2} x_{1} / 1+\left(1-g_{2}\right) g_{3} x_{2} / 1+\cdots$, the reciprocal of which is, except for an unimportant term and factor, the continued fraction (3.1). Theorem 3.1 is an improvement over Theorem 3.3, in that the $g_{n}$ 's after the first are permitted to be 0 . For this reason Theorem 3.1 contains the theorem given by Perron on page 258 (Theorem 26), in which it may be assumed with no loss in generality that $p_{1}>1$.

4. Totally monotone sequences corresponding to an "infinite distribution of mass." The sequence $c_{n}=\int_{0}^{1} u^{n} d \phi(u), n=0,1,2, \cdots$, in which $\phi(u)$ is real and monotone nondecreasing is completely characterized by the inequalities

$$
\Delta^{m} c_{n} \geqq 0, \quad m, n=0,1,2, \cdots,
$$

and is said to be a totally monotone sequence. If $\phi(u)$ has an infinite number of points of increase, we shall say that there is an infinite distribution of mass.

THEOREM 4.1. The sequence $c_{0}, c_{1}, c_{2}, \cdots$ is a totally monotone sequence corresponding to an infinite distribution of mass if and only if there exist real numbers $g_{1}, g_{2}, g_{3}, \cdots$ such that $0<g_{n}<1, n \geqq 1$, and such that

$$
\begin{aligned}
c_{0}-c_{1} x+c_{2} x^{2}-\cdots \sim c_{0} / 1 & +g_{1} x / 1+\left(1-g_{1}\right) g_{2} x / 1 \\
& +\left(1-g_{2}\right) g_{3} x / 1+\cdots, \quad\left(c_{0}>0\right) .
\end{aligned}
$$

Proof. Suppose that (4.2) holds. Then by Theorem 3.1 the continued fraction $1+g_{1} x / 1+\left(1-g_{1}\right) g_{2} x / 1+\left(1-g_{2}\right) g_{3} x / 1+\cdots$ converges uniformly for $|x| \leqq 1$. If $f(x)$ is the analytic function represented, then by Theorem 2.1 and Theorem 3.1, $(1+x) / f(x)$ is analytic for $|x|<1$, and therefore $c_{0} / f(x)$, the function represented by the continued fraction and series (4.2), is analytic for $|x|<1$.

Now the coefficients of $x$ in the continued fraction (4.2) are positive, and hence by the work of Stieltjes, this continued fraction represents a function of the form $\int_{0}^{\infty} d \phi(u) /(1+x u)$, where $\phi(u)$ is monotone nondecreasing, and has an infinite number of points of increase. Inasmuch as this function is analytic for $|x|<1$, and the corresponding continued fraction converges uniformly for $|x| \leqq r$ where $r$ is any positive number less than 1 , it follows that the upper limit of integration may be taken equal to 1 . Then $\Delta^{m} c_{n}=\int_{0}^{1}(1-u)^{m} u^{n} d \phi(u)$, and therefore (4.1) holds. Thus the sequence is totally monotone, and corresponds to an infinite distribution of mass.

Conversely, let $c_{n}=\int_{0}^{1} u^{n} d \phi(u)$, where $\phi(u)$ is monotone nondecreasing and has an infinite number of points of increase. That is, $c_{0}, c_{1}, c_{2}, \ldots$ is a totally

(7) See footnote 3. 
monotone sequence corresponding to an infinite distribution of mass. Then, by the work of Stieltjes, we must have a correspondence of the form

$$
c_{0}-c_{1} x+c_{2} x^{2}-\cdots \sim a_{1} / 1+a_{2} x / 1+a_{3} x / 1+\cdots,
$$

where $a_{1}, a_{2}, a_{3}, \cdots$ are real and positive. Moreover, the function represented by this series and continued fraction is $\int_{0}^{1} d \phi(u) /(1+x u)$. Since the limits of integration are from 0 to 1 , the zeros $\left({ }^{8}\right)$ of $B_{n}(x)$, the denominator of the $n$th approximant of the continued fraction, are real and less than -1 . Since $B_{n}(0)=1$ it therefore follows that $B_{n}(-1)>0$. We may then apply Theorem 2.3 , with $c=1$, to the continued fraction

$$
1+a_{2} x / 1+a_{3} x / 1+a_{4} x / 1+\cdots,
$$

the numerator of whose $n$th approximant is $B_{n+1}(x)$, and thus determine numbers $g_{1}, g_{2}, g_{3}, \cdots$, such that

$$
a_{2}=g_{1}, \quad a_{n}=g_{n-1}\left(1-g_{n-2}\right), \quad n>2 .
$$

Now, by Theorem 2.2,

$$
\Delta c_{0}-\Delta c_{1} x+\Delta c_{2} x^{2}-\cdots \sim \frac{\Delta c_{0}}{1}+\frac{g_{1}\left(1-g_{2}\right) x}{1}+\frac{g_{2}\left(1-g_{3}\right) x}{1}+\cdots .
$$

Also, $\Delta c_{n}=\int_{0}^{1} u^{n} d \phi_{1}(u)$ where $\phi_{1}(u)=\int_{0}^{u}(1-u) d \phi(u)$ is monotone nondecreasing and has an infinite number of points of increase. It follows that the coefficients of $x$ in the last continued fraction must all be positive, and that $\Delta c_{0}=c_{0}\left(1-g_{1}\right)>0$ (cf. (2.6)). We therefore have

$$
\begin{array}{rll}
g_{1}>0, & g_{n-1}\left(1-g_{n-2}\right)>0, & n>2, \\
1-g_{1}>0, & g_{n-2}\left(1-g_{n-1}\right)>0, & n>2,
\end{array}
$$

and consequently $0<g_{n}<1, n=1,2,3, \cdots$, as was to be proved.

5. Developments from the continued fraction algorithm. We shall begin by considering an example. It is known $\left({ }^{9}\right)$ that the series

$$
F(\alpha, 1, \gamma ;-x)=1-\frac{\alpha}{\gamma} x+\frac{\alpha(\alpha+1)}{\gamma(\gamma+1)} x^{2}-\cdots
$$

has the corresponding continued fraction $1+e_{1} x / 1+e_{2} x / 1+e_{3} x / 1+\cdots$, where

$e_{1}=\frac{\alpha}{\gamma}, \quad e_{2 n}=\frac{(\alpha+n)(\gamma+n-1)}{(\gamma+2 n-2)(\gamma+2 n-1)}, \quad e_{2 n+1}=\frac{n(\gamma+\alpha+n-1)}{(\gamma+2 n-1)(\gamma+2 n)}$.

Naturally $\alpha, \gamma$ are not negative integers or 0 . We then readily find that

(8) Perron, loc. cit., p. 368 and p. 383.

(9) Perron, loc. cit., p. 348. 


$$
F(\alpha, 1, \gamma ;-x)=\frac{1}{1}+\frac{g_{1} x}{1}+\frac{\left(1-g_{1}\right) g_{2} x}{1}+\frac{\left(1-g_{2}\right) g_{3} x}{1}+\cdots,
$$

where $g_{2 n}=n /(\gamma+2 n-1), g_{2 n-1}=(\alpha+n-1) /(\gamma+2 n-2), n=1,2,3, \cdots$ On applying Theorem 2.1 we readily obtain the power series identity

$$
F(\alpha, 1, \gamma ;-x)=\frac{1}{1+x}+\frac{\gamma-\alpha}{\gamma} \frac{x}{1+x} F(\alpha, 1, \gamma+1 ;-x)
$$

The repeated application of this identity gives the Euler expansion

$$
\begin{aligned}
F(\alpha, 1, \gamma ;-x)= & \frac{1}{1+x}+\frac{(\gamma-\alpha)}{\gamma} \frac{x}{(1+x)^{2}} \\
& +\frac{(\gamma-\alpha)(\gamma+1-\alpha)}{\gamma(\gamma+1)} \frac{x^{2}}{(1+x)^{3}}+\cdots
\end{aligned}
$$

We now propose to obtain the analogous developments for the general continued fraction of this form. For simplicity, let $g_{n}$ be real and $0<g_{n}<1$, and put

$$
f(x)=1+g_{1} x / 1+\left(1-g_{1}\right) g_{2} x / 1+\left(1-g_{2}\right) g_{3} x / 1+\cdots,
$$

so that

$$
(1+x) / f(x)=1+\left(1-g_{1}\right) x / 1+g_{1}\left(1-g_{2}\right) x / 1+g_{2}\left(1-g_{3}\right) x / 1+\cdots .
$$

Let $f_{1}(x)=1+g_{1}\left(1-g_{2}\right) x / 1+g_{2}\left(1-g_{3}\right) x / 1+\cdots$, and denote by $A_{n}(x)$ the numerator of the $n$th approximant of this continued fraction. Then it is easy to prove by mathematical induction that

$$
A_{n-1}(-1)=g_{1} g_{2} \cdots g_{n} S_{n},
$$

where

$$
S_{n}=1+\sum_{k=1}^{n} \frac{\left(1-g_{1}\right)\left(1-g_{2}\right) \cdots\left(1-g_{k}\right)}{g_{1} g_{2} \cdots g_{k}} .
$$

We may therefore apply Theorem 2.3 with $c=1$ and obtain

$$
f_{1}(x)=1+g_{1}^{(1)} x / 1+g_{2}^{(1)}\left(1-g_{1}^{(1)}\right) x / 1+g_{3}^{(1)}\left(1-g_{2}^{(1)}\right) x / 1+\cdots,
$$

where $g_{n}^{(1)}=g_{n}\left(1-g_{n+1}\right) A_{n-2}(-1) / A_{n-1}(-1)=\left(1-g_{n+1}\right) S_{n-1} / S_{n}, n=1,2,3, \cdots$, $\left(S_{0}=1\right)$. Consequently $0<g_{n}^{(1)}<1$, so that the continued fraction for $f_{1}(x)$ has precisely the same form as that for $f(x)$. Hence we may write

$$
\frac{1+x}{f_{1}(x)}=1+\frac{\left(1-g_{1}^{(1)}\right) x}{1}+\frac{g_{1}^{(1)}\left(1-g_{2}^{(1)}\right) x}{1}+\frac{g_{2}^{(1)}\left(1-g_{3}^{(1)}\right) x}{1}+\cdots
$$


put $f_{2}(x)=1+g_{1}^{(1)}\left(1-g_{2}^{(1)}\right) x / 1+g_{2}^{(1)}\left(1-g_{3}^{(1)}\right) x / 1+\cdots$, and apply the above argument to the latter continued fraction. In this manner we arrive at the following theorem:

Theorem 5.1. Let $f(x)=1+g_{1} x / 1+\left(1-g_{1}\right) g_{2} x / 1+\left(1-g_{2}\right) g_{3} x / 1+\cdots$, where $0<g_{n}<1$. Define numbers $g_{n}^{(m)}$ by the relations

$$
g_{k}^{(0)}=g_{k}, \quad g_{k}^{(m)}=\left(1-g_{k+1}^{(m-1)}\right) S_{k-1}^{(m-1)} / S_{k}^{(m-1)},
$$

$m, k=1,2,3, \cdots$,

$$
S_{0}^{(k)}=1, \quad S_{i}^{(k)}=1+\sum_{j=1}^{i} \frac{\left(1-g_{1}^{(k-1)}\right)\left(1-g_{2}^{(k-1)}\right) \cdots\left(1-g_{j}^{(k-1)}\right)}{g_{1}^{(k-1)} g_{2}^{(k-1)} \cdots g_{j}^{(k-1)}},
$$

so that $0<g_{n}^{(m)}<1 ;$ and put $f_{k}(x)=1+g_{1}^{(k)} x / 1+\left(1-g_{1}^{(k)}\right) g_{2}^{(k)} x / 1+\left(1-g_{2}^{(k)}\right) g_{3}^{(k)} x / 1$ $+\cdots$. Then the functions $f_{0} \equiv f, f_{1}, f_{2}, f_{3}, \cdots$ satisfy the following identities:

$$
\frac{1}{f_{k}}=\frac{1}{1+x}+\left(1-g_{1}^{(k)}\right) \frac{x}{1+x} \frac{1}{f_{k+1}}
$$

$k=0,1,2, \cdots$, and consequently $1 / f(x)$ has the Euler expansion

$$
\frac{1}{f(x)}=\frac{1}{1+x}+\left(1-g_{1}\right) \frac{x}{(1+x)^{2}}+\left(1-g_{1}\right)\left(1-g_{1}^{(1)}\right) \frac{x^{2}}{(1+x)^{3}}+\cdots
$$

Corollary 5.1. Let $c_{0} \neq 0$, and $c_{0} / f(x)=c_{0}-c_{1} x+c_{2} x^{2}-\ldots$. Then

$$
\Delta^{m} c_{0}=c_{0}\left(1-g_{1}\right)\left(1-g_{1}^{(1)}\right) \cdots\left(1-g_{1}^{(m-1)}\right) .
$$

COROLLARY 5.2. The correspondence

$$
\begin{aligned}
\Delta^{m} c_{0}-\Delta^{m} c_{1} x+\Delta{ }^{m} c_{2} x^{2}-\cdots \sim & \frac{\Delta^{m} c_{0}}{1}+\frac{g_{1}^{(m)} x}{1}+\frac{\left(1-g_{1}^{(m)}\right) g_{2}^{(m)} x}{1}-\frac{\left(1-g_{2}^{(m)}\right) g_{3}^{(m)} x}{1}+\cdots \\
+ & +\cdots
\end{aligned}
$$

is valid for $m=0,1,2, \ldots$.

From (5.4) it follows that if $c_{0}>0$ then $\Delta^{m} c_{0}>0$ for $m=1,2,3, \cdots$. We shall show next that $\Delta^{m} c_{n}>0$ for all $m, n$. To do this it will suffice to prove

THeOREM 5.2. If $0<g_{n}<1$ and $c_{0}-c_{1} x+c_{2} x^{2}-\cdots \sim c_{0} / 1+g_{1} x / 1+\left(1-g_{1}\right) g_{2} x / 1$

then

$$
+\left(1-g_{2}\right) g_{3} x / 1+\cdots,
$$


$c_{1}-c_{2} x+c_{3} x^{2}-\cdots \sim c_{1} / 1+h_{1} x / 1+\left(1-h_{1}\right) h_{2} x / 1$

where $0<h_{n}<1$.

$$
+\left(1-h_{2}\right) h_{3} x / 1+\cdots,
$$

Proof. With the aid of Stieltjes integrals and the results of $\$ 4$, one may prove this theorem in a few lines. The proof may be made by means of the continued fraction algorithm alone by means of the four lemmas which follow.

LEMMA 5.1. The continued fraction $\left({ }^{10}\right)$

$$
u_{1}+v_{1}-\frac{u_{1} v_{2}}{u_{2}+v_{2}}-\frac{u_{2} v_{3}}{u_{3}+v_{3}}-\cdots-\frac{u_{n-1} v_{n}}{u_{n}+v_{n}}
$$

in which $u_{i} \neq 0, i=1,2,3, \cdots, n$, is equal to

$$
v_{1}+\frac{u_{1}}{1+\frac{v_{2}}{u_{2}}+\frac{v_{2} v_{3}}{u_{2} u_{3}}+\cdots+\frac{v_{2} v_{3} \cdots v_{n}}{u_{2} u_{3} \cdots u_{n}}} .
$$

This may be readily proved by mathematical induction.

LEMMA 5.2. If $a_{n}>0$ and

$$
c_{0}-c_{1} x+c_{2} x^{2}-\cdots \sim a_{1} / 1+a_{2} x / 1+a_{3} x / 1+\cdots,
$$

then $\left({ }^{11}\right)$

$$
c_{1}-c_{2} x+c_{3} x^{2}-\cdots \sim b_{1} / 1+b_{2} x / 1+b_{3} x / 1+\cdots,
$$

where

$$
\begin{aligned}
b_{1} & =a_{1} a_{2}, \quad b_{2}=a_{2}+a_{3}, \quad b_{2 n+1}=a_{2 n+3}+a_{2 n+2}-b_{2 n+2}, \\
b_{2 n+2} & =a_{2 n+3}+\frac{a_{2 n+2}}{1+\frac{a_{2 n+1}}{a_{2 n}}+\frac{a_{2 n+1} a_{2 n-1}}{a_{2 n} a_{2 n-2}}+\cdots+\frac{a_{2 n+1} a_{2 n-1} \cdots a_{3}}{a_{2 n} a_{2 n-2} \cdots a_{2}}} .
\end{aligned}
$$

Proof. It is well known that $c_{1}-c_{2} x+c_{3} x^{2}-\cdots$ has a corresponding continued fraction of the form specified. Moreover, the "odd part" of $a_{1} / 1+a_{2} x / 1+a_{3} x / 1+\cdots$ must be the same as the "even part" of $b_{1} / 1+b_{2} x / 1+b_{3} x / 1+\cdots$. That is,

$$
\begin{aligned}
a_{1}- & \frac{a_{1} a_{2} x}{1+\left(a_{2}+a_{3}\right) x}-\frac{a_{3} a_{4} x^{2}}{1+\left(a_{4}+a_{5}\right) x}-\frac{a_{5} a_{6} x^{2}}{1+\left(a_{6}+a_{7}\right) x}-\cdots \\
& =c_{0}-\frac{b_{1} x}{1+b_{2} x}-\frac{b_{2} b_{3} x^{2}}{1+\left(b_{3}+b_{4}\right) x}-\frac{b_{4} b_{5} x^{2}}{1+\left(b_{5}+b_{6}\right) x}-\cdots .
\end{aligned}
$$

$\left.{ }^{(10}\right)$ O. Szász, Ueber die Erhaltung der Konvergenz unendlicher Kettenbr üche $\cdots$, Journal für die reine und angewandte Mathematik, vol. 147 (1916), pp. 132-160.

(11) This holds for any $a_{n}$ 's such that there is no division by zero in the formulas. 
On equating corresponding elements in these continued fractions we obtain (5.6) and the relation $b_{2 n} b_{2 n+1}=a_{2 n+1} a_{2 n+2}$. Hence, on combining this with (5.6),

$$
b_{2 n+2}=a_{2 n+3}+a_{2 n+2}-\frac{a_{2 n+1} a_{2 n+2}}{a_{2 n+1}+a_{2 n}}-\frac{a_{2 n-1} a_{2 n}}{a_{2 n-1}+a_{2 n-2}}-\cdots-\frac{a_{3} a_{4}}{a_{2}+a_{3}},
$$

which, by Lemma 5.1, reduces to (5.7).

LEMMA 5.3. If in Lemma 5.2 the $a_{n}$ 's have the form $a_{2}=g_{1}, a_{n}=g_{n-1}\left(1-g_{n-2}\right)$, $n>2$, where $0<g_{n}<1$, then $0<b_{n}<1, n \geqq 2$.

Proof. We have $0<b_{2}=1-\left(1-g_{1}\right)\left(1-g_{2}\right)<1, \quad 0<b_{2 n+2}=1-\left(1-g_{2 n+1}\right)$ $\cdot\left(1-g_{2 n+2}\right)-g_{2 n+1} g_{2 n}<1$. Hence also, $0<b_{2 n+1}<1$.

LEMMA 5.4. Under the hypothesis of Lemma 5.3 the numerators of the approximants of the continued fraction $1+b_{2} x / 1+b_{3} x / 1+b_{4} x / 1+\cdots$ are all positive for $x=-1$.

Proof. Let $A_{n}(x), n=0,1,2, \cdots$, be the numerators in question, and let $C_{n}(x)$ be the denominator of the $n$th approximant of

$$
a_{1} / 1+a_{2} x / 1+a_{3} x / 1+\cdots \text {. }
$$

Then by (5.8), $C_{2 n+1}(x) \equiv A_{2 n}(x)$. Now $\left|C_{2}(x)\right|=\left|1+g_{1} x\right| \geqq\left(1-g_{1}\right)\left|C_{1}(x)\right|$, if $|x| \leqq 1$. If, for any value of $n,|x| \leqq 1$ implies that

$$
\left|C_{n}(x)\right| \geqq\left(1-g_{n-1}\right)\left|C_{n-1}(x)\right| \geqq\left(1-g_{1}\right)\left(1-g_{2}\right) \cdots\left(1-g_{n-1}\right),
$$

then $\left|C_{n+1}(x)\right|=\left|C_{n}(x)+g_{n}\left(1-g_{n-1}\right) x C_{n-1}(x)\right| \geqq\left|C_{n}(x)\right|-g_{n}\left(1-g_{n-1}\right)\left|C_{n-1}(x)\right|$ if $|x| \leqq 1$. Hence $\left({ }^{12}\right)$

$$
\left|C_{n+1}(x)\right| \geqq\left(1-g_{n}\right)\left|C_{n}(x)\right| \geqq\left(1-g_{1}\right)\left(1-g_{2}\right) \cdots\left(1-g_{n}\right) .
$$

Inasmuch as $C_{n}(0)=1$, we must therefore have $C_{2 n+1}(-1)=A_{2 n}(-1)>0$. Now $A_{2 n+2}(x)=A_{2 n+1}(x)+b_{2 n+3} x A_{2 n}(x)$. Hence if $A_{2 n+1}(x)=0$ for $-1 \leqq x \leqq 0$, then $A_{2 n+2}(x)$ and $A_{2 n}(x)$ would have opposite signs for this value of $x$. Since this is impossible, it follows that $A_{2 n+1}(-1)>0$.

The proof of Theorem 5.2 may now be readily made. By Lemma 5.4 we may apply Theorem 2.3 with $c=1$ and obtain $b_{2}=h_{1}, b_{n}=h_{n-1}\left(1-h_{n-2}\right), n>2$, where $h_{n}=b_{n+1} A_{n-2}(-1) / A_{n-1}(-1)>0$. But by Lemma $5.3,0<h_{1}<1$, $0<h_{n-1}\left(1-h_{n-2}\right)<1, m>2$. Consequently $0<h_{n}<1, n \geqq 1$.

Theorem 5.3. If $c_{0}>0$, and

$$
\begin{aligned}
c_{0}-c_{1} x+c_{2} x^{2}-\cdots \sim c_{0} / 1+g_{1} x / 1+(1 & \left.-g_{1}\right) g_{2} x / 1 \\
& +\left(1-g_{2}\right) g_{3} x / 1+\cdots,
\end{aligned}
$$

where $0<g_{n}<1$, then

(12) This induction was used by Van Vleck, loà cit. 


$$
\Delta^{m} c_{n}>0
$$

$$
m, n=0,1,2, \cdots,
$$

and

$$
a<\frac{\Delta c_{n}}{c_{n}}<\frac{\Delta^{2} c_{n}}{\Delta c_{n}}<\frac{\Delta^{3} c_{n}}{\Delta^{2} c_{n}}<\cdots<1, \quad n=0,1,2, \cdots .
$$

Proof. The inequalities (5.10) follow from (5.4) and Theorem 5.2. It will suffice to prove (5.11) for the case $n=0$. By (5.4) we have

$$
\begin{aligned}
1 & >\frac{\Delta^{m+1} c_{0}}{\Delta^{m} c_{0}}=\left(1-g_{1}^{(m)}\right)=1-g_{1}^{(m-1)}\left(1-g_{2}^{(m-1)}\right) \\
& >1-g_{1}^{(m-1)}-\frac{\Delta^{m} c_{0}}{\Delta^{m-1} c_{0}}>0,
\end{aligned}
$$

$m=1,2,3, \cdots$, which was to be proved.

6. Totally monotone sequences corresponding to a finite distribution of mass. Consider a terminating continued fraction of the form $c_{0} / 1+g_{1} x / 1$ $+\left(1-g_{1}\right) g_{2} x / 1+\cdots+\left(1-g_{k-1}\right) g_{k} x / 1$, where $c_{0}>0,0<g_{n}<1, n=1,2,3, \cdots$, $k-1,0<g_{k} \leqq 1$. We know that if $k=2 m-1$ this continued fraction represents a rational function of the form

$$
\frac{A_{2 m}(x)}{B_{2 m}(x)}=\frac{M_{1}}{1+x x_{1}}+\frac{M_{2}}{1+x x_{2}}+\cdots+\frac{M_{m}}{1+x x_{m}},
$$

where $M_{i}>0, i=1,2,3, \cdots, m$, and $0<x_{1}<x_{2}<\cdots<x_{m} \leqq 1$. On the other hand, if $k=2 m$, the function represented has the form

$$
\frac{A_{2 m+1}(x)}{B_{2 m+1}(x)}=M_{0}+\frac{M_{1}}{1+x x_{1}}+\frac{M_{2}}{1+x x_{2}}+\cdots+\frac{M_{m}}{1+x x_{m}},
$$

where $M_{i}>0, i=0,1,2, \cdots, m$, and $0<x_{1}<x_{2}<\cdots<x_{m} \leqq 1$. Naturally the $M_{i}$ 's, $x_{i}$ 's are not the same in the two cases. In either case if $c_{0}-c_{1} x$ $+c_{2} x^{2}-\cdots$ is the corresponding power series, then

$$
c_{n}=\sum_{i=0}^{i=m} x_{i}^{n} M_{i}, \quad n=0,1,2, \cdots,
$$

where $x_{0}=0, x_{0}^{0}=1$, and $M_{0}$ is positive or 0 according as $k=2 m$ or $2 m-1$. Thus we may write $c_{n}=\int_{0}^{1} u^{n} d \phi(u), n=0,1,2, \cdots$, where $\phi(u)$ is a monotone nondecreasing function with but a finite number of points of increase.

Conversely, let $c_{0}, c_{1}, c_{2}, \cdots$ be a totally monotone sequence corresponding to a finite distribution of mass. Then $c_{n}$ has a representation of the form

$$
c_{n}=\int_{0}^{1} u^{n} d \phi(u), \quad n=0,1,2, \cdots,
$$


where $\phi(u)$ is a step-function. There are two cases to be considered according as $\phi(u)$ is or is not continuous at $u=0$.

Lemma 6.1. Let $A_{n}, B_{n}$ denote the determinants $\left|c_{i+j}\right|,\left|c_{i+j+1}\right| \quad(i, j=0$, $1, \cdots, n)$, respectively. Then if $\phi(u)$ is continuous at $u=0$, these determinants are positive for $n<m$, and if $\phi(u)$ is discontinuous at $u=0, A_{n}>0$ for $n \leqq m$, and $B_{n}>0$ for $n<m$, where $m$ is the number of values of $u>0$ where $\phi(u)$ is discontinuous.

Proof. Put

$$
c_{n}=\sum_{i=0}^{i=m} x_{i}^{n} M_{i}, \quad n=0,1,2, \cdots,
$$

where $0<x_{1}<x_{2}<\cdots<x_{m} \leqq 1, M_{1}, M_{2}, \cdots, M_{m}$ are positive, and $x_{0}=0$, $x_{0}^{0}=1$. If $\phi(u)$ is continuous at $u=0$, then $M_{0}=0$, while if $\phi(u)$ is discontinuous at $u=0, M_{0}>0$. Consider the quadratic form

$$
\sum_{i, j=0}^{n} c_{i+j+r} \xi_{i} \xi_{j}=\int_{0}^{1} u^{r}\left\{\sum_{i=0}^{i=n} \xi_{i} u^{i}\right\}^{2} d \phi(u) .
$$

If $r=1$ this is clearly positive definite for $n<m$, so that $B_{n}>0$ for $n<m$ in both cases. When $r=0$, it is positive definite for $n<m$ if $\phi(u)$ is continuous at $u=0$, and for $n \leqq m$ if $\phi(u)$ is discontinuous at $u=0$. Hence $A_{n}>0$ in the first case for $n<m$, and in the second for $n \leqq m$.

Now

$$
\begin{aligned}
P(x) \equiv c_{0}-c_{1} x+c_{2} x^{2}-\cdots \equiv M_{0} & +\frac{M_{1}}{1+x x_{1}}+\frac{M_{2}}{1+x x_{2}}+\cdots \\
& +\frac{M_{m}}{1+x x_{m}}=\frac{S(x)}{T(x)},
\end{aligned}
$$

where $T(x)$ is a polynomial of degree $m$, and $S(x)$ is of degree $m$ or $m-1$ according as $M_{0}>0$ or $M_{0}=0$. Put

$$
a_{1}=c_{0}, \quad a_{2 n}=B_{n-1} A_{n-2} / A_{n-1} B_{n-2}, \quad a_{2 n+1}=A_{n} B_{n-2} / B_{n-1} A_{n-1},
$$

$n=1,2,3, \cdots, A_{-1}=B_{-1}=1$, and form the continued fractions

$$
\frac{A_{2 m}(x)}{B_{2 m}(x)}=\frac{a_{1}}{1}+\frac{a_{2} x}{1}+\cdots+\frac{a_{2 m} x}{1}+\frac{0 \cdot x}{1},
$$

and

$$
\frac{A_{2 m+1}(x)}{B_{2 m+1}(x)}=\frac{a_{1}}{1}+\frac{a_{2} x}{1}+\cdots+\frac{a_{2 m+1} x}{1}+\frac{0 \cdot x}{1},
$$

according as $M_{0}=0$ or $M_{0}>0$. The vanishing partial quotient is affixed for a 
reason to appear presently. On taking account of the degrees of numerators and denominators, and of the degree of approximation of these rational fractions to the power series $P(x)$, we conclude that they are identical with $S(x) / T(x)$ in the two cases.

The denominators of the $n$th approximants of (6.2), (6.3) are greater than 0 if $-1 \leqq x \leqq 0$, and $n<2 m, n<2 m+1$, respectively. Hence we may apply Theorem 2.3 with $c=1$ to show that the $a_{n}$ 's have the form $g_{n-1}\left(1-g_{n-2}\right)$, $n>2, a_{2}=g_{1}$. We then apply Theorem 2.2 and obtain in the case of (6.2):

$$
\begin{aligned}
\Delta c_{0}-\Delta c_{1} x+\Delta c_{2} x^{2}-\cdots \sim \frac{\Delta c_{0}}{1} & +\frac{g_{1}\left(1-g_{1}\right) x}{1}+\cdots \\
& +\frac{g_{2 m-2}\left(1-g_{2 m-1}\right) x}{1}+\frac{g_{2 m-1} x}{1} .
\end{aligned}
$$

It is easy to see that $\phi(u)$ is discontinuous at $u=1$ if and only if $g_{2 m-1}=1$. In case $g_{2 m-1}=1$, the above continued fraction terminates with the $(2 m-2)$ th partial quotient, while if $g_{2 m-1}<1$ it terminates with the $2 m$ th partial quotient.

Now $\Delta c_{n}=\int_{0}^{1} u^{n} d \phi_{1}(u)$, where $\phi_{1}(u)=\int_{0}^{u}(1-u) d \phi(u)$. Hence $\phi_{1}(u)$ has the same number of discontinuities as, or a smaller number by one than, $\phi(u)$, according as 1 is not or is, respectively, a point of discontinuity of $\phi(u)$. Since $\phi_{1}(u)$ is a function of the same character as $\phi(u)$, we conclude that $1-g_{1}>0$, $g_{n-1}\left(1-g_{n}\right)>0, n=2,3, \cdots, 2 m-2$ if $g_{2 m-1}=1$, and $n=2,3, \cdots, 2 m-1$ if $g_{2 m-1}<1$. But we previously had $g_{1}>0, g_{n}\left(1-g_{n-1}\right)>0, n=2,3, \cdots, 2 m-1$. Hence we conclude that $0<g_{n}<1, n=1,2,3, \cdots, 2 m-2,0<g_{2 m-1} \leqq 1$. The treatment of $(6.3)$ is exactly the same. We have therefore completed the proof of the following theorem:

THEOREM 6.1. If $c_{0}, c_{1}, c_{2}, \cdots$ is a totally monotone sequence corresponding to a finite distribution of mass, then $c_{0}-c_{1} x+c_{2} x^{2}-\cdots$ is the constant $c_{0} \geqq 0$, or else

$$
\begin{aligned}
c_{0}-c_{1} x+c_{2} x^{2}-\cdots \sim c_{0} / 1+g_{1} x / 1+\left(1-g_{1}\right) g_{2} x / 1 & +\cdots \\
& +g_{k}\left(1-g_{k-1}\right) x / 1,
\end{aligned}
$$

where $0<g_{n}<1, n<k, 0<g_{k} \leqq 1, c_{0}>0$. Conversely, any sequence determined in this way is a totally monotone sequence corresponding to a finite distribution of mass.

7. The moment problem for the interval $(-\infty, 1)$. The methods used previously may be employed to prove the following theorem:

THEOREM 7.1. If $\phi(u)$ is a monotone nondecreasing function in the interval $(-\infty, 1)$, such that the moments $c_{n}=\int_{-\infty}^{1} u^{n} d \phi(u), n=0,1,2, \cdots$, are all finite, and if the series $c_{0}-c_{1} x+c_{2} x^{2}-\cdots$ has a corresponding continued fraction, 
then this continued fraction must have the form

$$
c_{0} / 1+g_{1} x / 1+\left(1-g_{1}\right) g_{2} x / 1+\left(1-g_{2}\right) g_{3} x / 1+\cdots,
$$

where $c_{0}>0$, and

$$
g_{2 n-1} g_{2 n}>0, \quad\left(1-g_{2 n-1}\right)\left(1-g_{2 n-2}\right)>0,
$$

$n=1,2,3, \cdots, g_{0}=0$.

Proof. Let $b_{1} / 1+b_{2} x / 1+b_{3} x / 1+\cdots$ be the corresponding continued fraction which is supposed to exist. Then it is known $\left({ }^{13}\right)$ that $b_{1}>0, b_{2 n} b_{2 n+1}>0$, $n=1,2,3, \cdots$. The zeros of the denominators $B_{n}(x)$ of the approximants of this continued fraction are all real and none of them lie in the interval $\left({ }^{14}\right)$ $-1 \leqq x \leqq 0$. Hence $B_{n}(-1)>0$. By Theorem 2.3 we may then write this continued fraction in the form (7.1) where $g_{n} \neq 0,1, n \geqq 1$. Then by Theorem 2.2 we have

$$
\Delta c_{0}-\Delta c_{1} x+\Delta c_{2} x^{2}-\cdots \sim \frac{\Delta c_{0}}{1}+\frac{g_{1}\left(1-g_{2}\right) x}{1}+\frac{g_{2}\left(1-g_{3}\right) x}{1}+\cdots .
$$

Now $\Delta^{m} c_{n}=\int_{-\infty}^{1}(1-u)^{m} u^{n} d \phi(u)=\int_{-\infty}^{1} u^{n} d \phi_{1}(u)$, where $\phi_{1}(u)=\int_{-\infty}^{u}(1-u)^{m} d \phi(u)$ is monotone nondecreasing for $-\infty<u \leqq 1$, and consequently $\Delta c_{0}=c_{0}\left(1-g_{1}\right)$ $>0, g_{2 n-1} g_{2 n}\left(1-g_{2 n}\right)\left(1-g_{2 n+1}\right)>0, n \geqq 1$. The theorem now follows. We shall leave unanswered the question of the converse of this theorem.

8. Moment generating functions which are bounded in the unit circle. The problem of this section is to specialize the continued fractions of Theorems 4.1 and 6.1 in such a way that the moment generating function $f(x)=c_{0}-c_{1} x+c_{2} x^{2}-\cdots$ will be bounded in the unit circle, that is

$$
M(f)=\underset{|x|<1}{\operatorname{l.u.b.}}|f(x)|
$$

will be finite. Our first result is contained in the theorem which follows.

THEOREM 8.1. The function $f(x)=c_{0}-c_{1} x+c_{2} x^{2}-\cdots$ is in the class $E$, of moment generating functions bounded in the unit circle, if and only if there is a correspondence of the form

$$
h f(x) \sim g_{1} / 1+\left(1-g_{1}\right) g_{2} x / 1+\left(1-g_{2}\right) g_{3} x / 1+\cdots,
$$

for some sufficiently small positive number $h$ and for real $g_{n}$ 's such that $0 \leqq g_{n} \leqq 1$, $n=1,2,3, \cdots$, where it is agreed that the continued fraction shall terminate with the first identically vanishing partial quotient. When the condition is satisfied, $M(f) \leqq 1 / h$, and $h$ can be taken equal to 1 if and only if $M(f) \leqq 1$.

Proof of sufficiency. Suppose first that the continued fraction terminates

(13) H. Hamburger, Mathematische Annalen, vol. 81 (1920), pp. 235-319, and vol. 82 (1921), pp. 120-187.

(14) Cf. footnote 8 . 
and that $h f(x)=g_{1}, 0 \leqq g_{1} \leqq 1$, or $h f(x)=g_{1} / 1+\left(1-g_{1}\right) g_{2} x / 1+\cdots+\left(1-g_{k}\right)$ $\cdot g_{k+1} x / 1, k \geqq 1,0<g_{n}<1, n=1,2,3, \cdots, k, 0<g_{k+1} \leqq 1$. The first possibility is at once disposed of. When $g_{k+1}<1$, the second possibility is disposed of by Theorem 3.1; and when $g_{k+1}=1$, it is required to be shown that the rational function $h f(x)$ has no poles for $|x| \leqq 1$. To do this, recall that the function $1 /(1+x h f(x))$ has the form

$$
\frac{M_{0}}{1+x x_{0}}+\frac{M_{1}}{1+x x_{1}}+\cdots+\frac{M_{m-1}}{1+x x_{m-1}}+\frac{M_{m}}{1+x x_{m}}
$$

where $x_{0}=0<x_{1}<x_{2}<\cdots<x_{m} \leqq 1, M_{0} \geqq 0, M_{i}>0, i=1,2, \cdots, m$, and that in the case under consideration $x_{m}=1$. Consequently, the zero of this function which lies nearest the origin is less than -1 , so that the function $1+x h f(x)$ has no pole for $|x| \leqq 1$ and therefore $M(f)$ is finite. It will be seen that $f(-1)=1 / h=c_{0}+c_{1}+c_{2}+\cdots=M(f)$.

When the continued fraction does not terminate, then by Theorem 3.1, $M(f) \leqq 1 / h$, and $f(x) \varepsilon E$.

Proof of necessity. Suppose conversely that $f(x) \varepsilon E$, and let $f(x)=c_{0} / 1$ $+h_{1} x / 1+\left(1-h_{1}\right) h_{2} x / 1+\left(1-h_{2}\right) h_{3} x / 1+\cdots$, where $c_{0} \geqq 0,0 \leqq h_{n} \leqq 1, n \geqq 1$. If $c_{0}=0$ or $h_{1}=0$, the theorem is obviously true. Suppose $c_{0}>0,0<h_{n}<1$, $n=1,2, \cdots, k-1,0<h_{k} \leqq 1$, and that the continued fraction terminates by having $h_{k+1}\left(1-h_{k}\right)=0$. Then we must have $h_{k}<1$, for otherwise $f(x)$ has a pole at $x=-1$. Now consider, whether or not the continued fraction terminates, the function

$$
\begin{aligned}
1 /(1+x h f(x))= & 1 / 1+h c_{0} x / 1+h_{1} x / 1+\left(1-h_{1}\right) h_{2} x / 1 \\
& +\left(1-h_{2}\right) h_{3} x / 1+\cdots .
\end{aligned}
$$

Since $0<h_{1}<1,0 \leqq h_{n}<1, n>1$, the continued fraction $1+h_{1} x / 1+\left(1-h_{1}\right) h_{2} x / 1$ $+\left(1-h_{2}\right) h_{3} x / 1+\cdots$ converges uniformly for $|x| \leqq 1$, by Theorem 3.1 , and cannot vanish for $|x| \leqq 1$ since $M(f)<\infty$ by hypothesis. It readily follows that $h>0$ can be so chosen $(h=1$ if $M(f) \leqq 1)$, that for every $r, 0<r<1$, the continued fraction for $1 /(1+x h f(x))$ converges uniformly for $|x| \leqq r$, and consequently we must have, for the chosen value of $h$,

$$
1 /(1+x h f(x))=\int_{0}^{1} d \phi(u) /(1+x u)
$$

where $\phi(u)$ is some monotone nondecreasing function. Hence $1 /(1+x h f(x))$ is a moment generating function, and therefore has a representation of the form $1 / 1+g_{1} x / 1+\left(1-g_{1}\right) g_{2} x / 1+\left(1-g_{2}\right) g_{3} x / 1+\cdots$, so that $h f(x)$ has a representation as prescribed by the theorem.

Another characterization of $E$ in terms of continued fractions is given by the theorem which follows: 
THEOREM 8.2. Given a moment generating function $f(x)=c_{0} / 1+g_{1} x / 1$ $+\left(1-g_{1}\right) g_{2} x / 1+\cdots\left(c_{0} \geqq 0,0 \leqq g_{n} \leqq 1\right), f(x) \varepsilon E$ if and only if $f(x) \equiv c_{0} \geqq 0$, or $f(x)=c_{0} / 1+g_{1} x / 1+\left(1-g_{1}\right) g_{2} x / 1+\cdots+\left(1-g_{k-2}\right) g_{k-1} x / 1$, where $c_{0}>0$, $0<g_{n}<1, n=1,2,3, \cdots, k-1$; or else $f(x)=c_{0} / 1+g_{1} x / 1+\left(1-g_{1}\right) g_{2} x / 1$ $+\left(1-g_{2}\right) g_{3} x / 1+\cdots$, where $c_{0}>0,0<g_{n}<1, n \geqq 1$, and the series

$$
1+\sum_{n=1}^{n=\infty} \frac{g_{1} g_{2} \cdots g_{n}}{\left(1-g_{1}\right)\left(1-g_{2}\right) \cdots\left(1-g_{n}\right)}
$$

converges.

Proof. The case of the terminating continued fraction is easily disposed of, for when the condition is satisfied the rational function has no poles for $|x| \leqq 1$, while if $g_{k-1}=1$ (the only alternative) there is a pole at $x=-1$.

In the case of the nonterminating continued fraction, the function $c_{0} / f(x)=1+g_{1} x / 1+\left(1-g_{1}\right) g_{2} x / 1+\left(1-g_{2}\right) g_{3} x / 1+\cdots$ is analytic for $|x|<1$ and continuous for $|x| \leqq 1$. Also by Theorem 2.1 the function $(1+x) f(x)$ enjoys these same properties. Thus $f(x)$ is continuous for $|x| \leqq 1$ except possibly at $x=-1$. But by Theorem $3.1 f(-1) / c_{0}$ is equal to the series $(8.1)$ and is therefore finite if and only if the latter converges.

9. A characterization of $E$ in terms of the moments $c_{n}$. We now prove

Theorem 9.1. A moment generating function $f(x)=c_{0}-c_{1} x+c_{2} x^{2}-\cdots$ is in $E$ if and only if the series $c_{0}+c_{1}+c_{2}+\cdots$ converges, and when the condition is satisfied we have

$$
M(f)=c_{0}+c_{1}+c_{2}+\cdots .
$$

Proof. Sufficiency: When the series $c_{0}+c_{1}+c_{2}+\cdots$ converges, the sequence $d_{n}=c_{n}+c_{n+1}+c_{n+2}+\cdots, n=0,1,2, \cdots$, is totally monotone, so that the power series $d_{0}-d_{1} x+d_{2} x^{2}-\cdots$ has a corresponding continued fraction of the form $d_{0} / 1+h_{1} x / 1+\left(1-h_{1}\right) h_{2} x / 1+\left(1-h_{2}\right) h_{3} x / 1+\cdots$, $\left(d_{0} \geqq 0,0 \leqq h_{n} \leqq 1\right)$. Then, since $\Delta d_{n}=c_{n}$, it follows from Theorem 2.2 that $c_{0}-c_{1} x+c_{2} x^{2}-\cdots \sim c_{0} / 1+\left(1-g_{1}\right) g_{2} x / 1+\left(1-g_{2}\right) g_{3} x / 1+\cdots$ where we have put $g_{n}=1-h_{n}, n=1,2,3, \cdots$. That $f(x) \varepsilon E$ now follows from Theorem 8.1.

Necessity: The necessity of the condition results at once from a theorem of Abel. However, it is interesting to give a direct proof. Suppose then that $f(x) \varepsilon E$. Then by Theorem 8.1 we must have $h f(x) \sim g_{1} / 1+\left(1-g_{1}\right) g_{2} x / 1$ $+\left(1-g_{2}\right) g_{3} x / 1+\cdots$ for some $h>0$, where the $g_{n}$ 's are restricted as in Theorem 8.1. Therefore $1+h x f(x) \sim 1+g_{1} x+\left(1-g_{1}\right) g_{2} x / 1+\left(1-g_{2}\right) g_{3} x / 1+\cdots$, so that, by Theorem 2.1 ,

$$
\frac{1+h x f(x)}{1+x} \sim \frac{1}{1}+\frac{\left(1-g_{1}\right) x}{1}+\frac{g_{1}\left(1-g_{2}\right) x}{1}+\cdots .
$$

Consequently the function $(1+h x f(x)) /(1+x)$ is a moment generating func- 
tion. Its power series expansion is $1-\left(1-h c_{0}\right) x+\left(1-h c_{0}-h c_{1}\right) x^{2}-\cdots$, so that $1-h\left(c_{0}+c_{1}+c_{2}+\cdots+c_{n}\right) \geqq 0$ for $n=0,1,2, \cdots$. It follows that the series $\sum c_{i}$ is convergent.

Since $f(-1)=c_{0}+c_{1}+c_{2}+\cdots$, it follows that $M(f)$ is equal to this sum.

As a corollary to Theorem 9.1 we have

THEOREM 9.2. The moment generating function $f(x)$ is in $E$ if and only if it has a Stieltjes integral representation of the form

$$
f(x)=\int_{0}^{1} \frac{(1-u) d \phi(u)}{1+x u},
$$

in which $\phi(u)$ is bounded and monotone nondecreasing.

Proof. If $f(x) \varepsilon E$, then the series $\sum c_{i}$ converges. Let $d_{n}$ be defined as in the proof of Theorem 9.1. Then the function $d_{0}-d_{1} x+d_{2} x^{2}-\cdots$ has a representation of the form $\int_{0}^{1} d \phi(u) /(1+x u)$, where $\phi(u)$ is bounded and monotone nondecreasing. Since we must have $f(x)=\int_{0}^{1}(1-u) d \phi(u) /(1+x u)$, the necessity of the condition is proved. Conversely, if the condition is fulfilled, put $d_{0}-d_{1} x+d_{2} x^{2}-\cdots=\int_{0}^{1} d \phi(u) /(1+x u)$, where $\phi(u)$ is given, Then $c_{n}=\Delta d_{n}$, so that $\sum c_{i}=d_{0}-\lim _{n=\infty} d_{n}$ is convergent, and consequently $f(x) \varepsilon E$.

10. The algorithm of Schur. Starting with a function $f(x)$ of $E$ for which $M(f) \leqq 1$, we construct with $\operatorname{Schur}\left({ }^{15}\right)$ a sequence of functions $f_{n}(x)$ in the following way. Put $f_{0}(x)=f(x)$,

$$
f_{n+1}(x)=\frac{1}{x} \frac{t_{n}-f_{n}(x)}{1-t_{n} f_{n}(x)}, \quad t_{n}=f_{n}(0), \quad n=0,1,2, \cdots .
$$

If $M(f)=1$, then $M\left(f_{n}\right)=1$, and if $M(f)<1$, then $M\left(f_{n}\right)<1,(n=1,2,3, \cdots)$.

If $f(x) \equiv g_{1}$, then $f_{n}(x) \equiv 0$ for $n=1,2,3, \cdots$ If $f(x)=g_{1} / 1+\left(1-g_{1}\right) g_{2} x / 1$, then $f_{1}(x)=g_{1}^{(1)} / 1+\left(1-g_{1}^{(1)}\right) g_{2}^{(1)} x / 1$, where

$$
g_{1}^{(1)}=g_{1} g_{2} /\left(1+g_{1}\right), \quad g_{2}^{(1)}=g_{1} g_{2} /\left(1+g_{1}-g_{1} g_{2}\right),
$$

so that if $0<g_{1}<1,0<g_{2} \leqq 1$, then $0<g_{1}^{(1)}<1,0<g_{2}^{(1)} \leqq 1$. We shall prove this theorem:

THEOREM 10.1. If $f(x)=g_{1} / 1+\left(1-g_{1}\right) g_{2} x / 1+\left(1-g_{2}\right) g_{3} x / 1+\cdots, 0 \leqq g_{n} \leqq 1$, $n=1,2,3, \cdots$, with the agreement that the continued fraction shall terminate with the first identically vanishing partial numerator, so that $f(x)$ is a moment generating function and $M(f) \leqq 1$, then the functions $f_{1}(x), f_{2}(x), f_{3}(x), \cdots$ given by (10.1) are all moment generating functions and $M\left(f_{n}\right) \leqq 1$. The only case where any of these functions are constants is where $f(x) \equiv g_{1}$, whereupon $f_{n}(x) \equiv 0$, $n=1,2,3, \cdots$.

Proof. By (10.1), if $f(x)$ is not a constant, then

(15) Cf. footnote 4. 


$$
\begin{aligned}
x f_{1}(x) & =g_{1}-\frac{\left(1-g_{1}^{2}\right)}{-g_{1}+1 / f(x)} \\
& =g_{1}-g_{1} / 1+g_{2}\left(1-h_{1}\right) x / 1+g_{3}\left(1-g_{2}\right) x / 1+\cdots,
\end{aligned}
$$

where we have put $1-h_{1}=1 /\left(1+g_{1}\right), h_{1}=g_{1} /\left(1+g_{1}\right)$. This function is evidently of the form $g_{1}-g(x)$, where $g(x)$ is a moment generating function and $g(0)=g_{1}$. Put $g(x)=g_{1}-d_{0} x+d_{1} x^{2}-\cdots$, and we find that $f_{1}(x)=d_{0}-d_{1} x$ $+d_{2} x^{2}-\cdots$ and is therefore a moment generating function. On account of the character of the transformation used, $M\left(f_{1}\right) \leqq 1$. Consequently, by Theorem 8.1, $f_{1}(x)=g_{1}^{(1)} / 1+\left(1-g_{1}^{(1)}\right) g_{2}^{(1)} x / 1+\left(1-g_{2}^{(1)}\right) g_{3}^{(1)} x / 1+\cdots$ where $0 \leqq g_{n}^{(1)} \leqq 1, n=1,2,3, \cdots$, with the of $\mathrm{t}$ repeated convention regarding termination of the continued fraction.

In the same way, starting with $f_{1}(x)$ instead of with $f(x)$, we find that $f_{2}(x)$ is a moment generating function, and $M\left(f_{2}\right) \leqq 1$; and by induction, $f_{3}(x), f_{4}(x), \cdots$ all have this property.

We saw previously that when $f(x)=g_{1}$, then $f_{n}(x)=0,(n=1,2,3, \cdots)$. It remains to be shown that this is the only case where any of the functions can reduce to a constant. To do this, it suffices to show that if $f_{1}(x) \equiv c$, a constant, then $c=0$. We have

$$
f(x)=\frac{g_{1}-c x}{1-c x g_{1}}=g_{1}-\left(1-g_{1}^{2}\right) c x-g_{1} c^{2}\left(1-g_{1}^{2}\right) x^{2}-\cdots .
$$

Since this is a moment generating function, we must have $-g_{1} c^{2}\left(1-g_{1}^{2}\right) \geqq 0$, which implies that $c=0$, or else $g_{1}=0$ or 1 . But when $g_{1}=0$ or 1 , we must have $f=g_{1}$, and consequently $f_{1}=c=0$ in this case also.

As a corollary we have

THEOREM 10.2. If $f(x)$ is a moment generating function for which $M(f) \leqq 1$, then the sequence $t_{0}, t_{1}, t_{2}, \cdots$ given by (10.1) has the property of a totally monotone sequence that if any member is 0 the others are also 0 with the possible exception of the first.

In a number of examples which we have examined, the sequence $\left\{t_{n}\right\}$ has been found to be totally monotone. Technical difficulties have thus far prevented us from determining whether or not this is always the case. Also, the question as to the converse of this naturally arises, namely, if $\left\{t_{n}\right\}$ is a totally monotone sequence, will the function $f(x)$ which this sequence determines be a moment generating function with $M(f) \leqq 1$ ?

In conclusion we shall give recursion formulas for computing the $t_{n}$ 's in terms of the $g_{n}$ 's. From (10.2) we have

$$
x f_{1}(x)=g_{1}-\frac{g_{1}}{1}+\frac{\left(1-h_{1}\right) g_{2} x}{1}+\frac{\left(1-g_{2}\right) g_{3} x}{1}+\cdots,
$$


which is equal to $g_{1}^{(1)} x / 1+\left(1-g_{1}^{(1)}\right) g_{2}^{(1)} x / 1+\left(1-g_{2}^{(1)}\right) g_{3}^{(1)} x / 1+\cdots$. On equating the odd part of the first of these continued fractions to the even part of the second we obtain at once the formulas

$$
\begin{gathered}
g_{1}^{(1)}=g_{1} g_{2} /\left(1+g_{1}\right), \quad g_{2}^{(1)}\left(1-g_{1}^{(1)}\right)=\frac{g_{2}}{1+g_{1}}+g_{3}\left(1-g_{2}\right), \\
g_{n}^{(1)} g_{n+1}^{(1)}\left(1-g_{n-1}^{(1)}\right)\left(1-g_{n}^{(1)}\right)=g_{n+1} g_{n+2}\left(1-g_{n}\right)\left(1-g_{n+1}\right), \\
g_{n+1}^{(1)}\left(1-g_{n}^{(1)}\right)+g_{n+2}^{(1)}\left(1-g_{n+1}^{(1)}\right)=g_{n+2}\left(1-g_{n+1}\right)+g_{n+3}\left(1-g_{n+2}\right),
\end{gathered}
$$

$n=2,4,6, \cdots$. By means of these relations one may show that the sequence $t_{n}=g_{1}^{(n)}, n=0,1,2, \ldots\left(g_{1}^{(0)}=g_{1}\right)$ is monotone decreasing. In fact, $t_{n}<t_{n-1} /\left(1+t_{n-1}\right), n=1,2,3, \cdots$.

Using a result of Schur $\left({ }^{16}\right)$ one may obtain formulas for the $t_{n}$ 's in terms of the $g_{n}$ 's. To do this, let the $n$th approximant of the continued fraction for $f(x)$ be

$$
\frac{A_{n}(x)}{B_{n}(x)}=\frac{a_{0}+a_{1} x+a_{2} x^{2}+\cdots+a_{k} x^{k}}{b_{0}+b_{1} x+b_{2} x^{2}+\cdots+b_{r} x^{r}}
$$

This rational function is a moment generating function, and its modulus is less than or equal to 1 for $|x| \leqq 1$ provided $M(f) \leqq 1$. Moreover, the sequence $\left\{t_{i}\right\}$ for this function will be in agreement with that for $f(x)$ up to and including the term of index $n-1$. Moreover, the $a_{i}$ 's and $b_{i}$ 's can be computed in terms of the $g_{i}$ 's. Using the notation of Schur, we then put

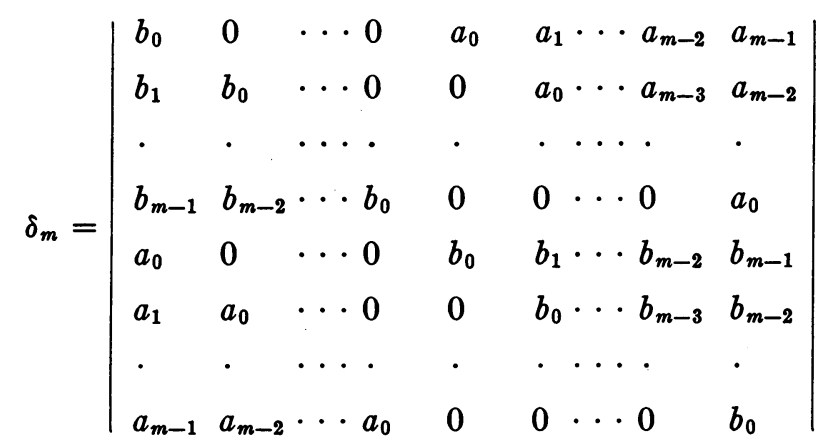

where $a_{i}=0$ if $i>k$ and $b_{i}=0$ if $i>r$. A formula of Schur $\left({ }^{17}\right)$ then gives for $t_{i}$

$$
t_{i}=\left\{1-\frac{\delta_{i-1} \delta_{i+1}}{\delta_{i}^{2}}\right\}^{1 / 2},
$$

which is valid for $i=0,1,2, \cdots, n-1$.

(16) Schur, loc. cit. (first part), pp. 213-215.

(17) Loc. cit., p. 215 , formula (12).

NORTHWESTERN UNIVERSITY,

Evanston, Ill. 